backward stroke, so that the diagram was di-torted and shortened, the drum being uniformly behind its proper position during the forward stroke, and before its position during the backward stroke. This distortion diminished the area of the diagram according to the rate of expansion and the length and elasticity of the cord ased. This was definitely expressed by a formula. This disturbance, the influence of which was very great in cases of high expansion, large engines, and ordinary cords, appeared to have been unnoticed. The circumstances on which it depended were the elasticity of the cord and the friction of the drum, and the question was how far these existed in the ordinary indicators. It might be said that the diagrams which led to the discovery of this effect were taken with an indicator which had been in constant use for several years. It was in apparently perfect condition, and the diagrams did not differ essentially from those which had been previously taken. The cord was one which had been sup slied by the maker. The manner of the discovery was described: For years the autbor had pursued in the cla $s$ the method of testing the vibrations of the indicator pencil by projecting them on to the crank-circle, and he had noticed that the first oscillation fell short, and shorter in the back diagram than in the front. The cause of this was not obvious, and it was partly with a view to determine this cause that Mr. Brightmore's investigation was commenced. A slight error in the reducing rod, which had a fixed centre and a slot in which a stud in the slide-block worked, was altered. This, however, did not get rid of the effect. A new cord was substituted for the old one, and the effect was found to be much enhanced, the new cord being more elastic than the old one. This reduced it to the stretching of the cord, but it was only after carefully working out the effect of the inertia of the drum, and it was seen this was to lengthen the first oscillation at the back end that the friction was examined. The indicator was taken to pieces, cleaned and oiled; then the effect wos much reduced. Several new wires and cords were used, and eventually steel wire was adopted as the best. The test supplied by the oscillations could only be applied to diagrams taken at high speeds, and the test furnished by the influence upon area was vague. What was wanted was an independent means of determining the simultaneous positions of the drum and the engine-piston. As the best method of meeting this, it was decided to arrange an electric circuit through the pencil to the drum, with sufficient electromotive force to prick the paper, making the engine-piston close this circuit at eleven definite equidistant points in the motion backwards and forwards. This was successfully carried out, and the stretching of the cord during the backward and forward strokes was definitely ascertained. Taking the smallest results obtained with a cord, it appeared from these experiments that the least difference of stretching was to make this difference in inches 5 per cent. of the length of the cord in feet. Examples of this effect in diminishing the mean indicated pressure were given. 'Thus, in a locomotive cutting off at one-quarter it was 8 per cent.; in a condensing engine having 3.5 feet stroke, cutting off at one-tenth, 20 per cent. ; and the same compounded, ro per cent.

These would seem to be the smallest results that could have occurred in ordinary practice. The conclusion, however, that hitherto the normal indicated power from engines had been from Io to 20 per cent. too small must wait for verification. Yet there were not wanting independent evidences of such an effect. In diagrams taken from engines at high speeds the admission line would not but for this effect be vertical. It would show a certain amount of detail, and the first oscillation would not have a sharp top. Noreover, it was commonly found that the expansion line, allowing for clearance, was above the true expansion line for the steam. This apparent rise in the curve of expansion was exactly what would result if the apparent cut-off was too early, and thi was the result of the effect that had been considered. The author had tried several diagrams, and found that after correction the expansion line came out very close to the true curve.

In making these comparisons the explanation of another feature of diagrams became apparent. When the two diagrams were traced on the same card, there was sometimes a want of symmetry about them, and in this case the cut-off was shorter on the back than on the front diagram. This the author attributed to the friction of the drum when the cord for the back diagram was longer than that for the front. When this was the case the relative lengths of the cord were about $I$ to $I \cdot 8$. These observations were illustrated in a diagram from "Richards" Indicator." To test this diagram a tracing was taken, and reversed so that the tront diagram was superimposed on the back. It was observed that the diagrams were of different lengths, and the difference was about the same as the difference in cut-off; that notwithstanding the apparent cut-off in the back diagram was to that in the front in the ratio of 2 to 3 , the expansion line of the back diagram was the same shape as that. in the front; and that if the diagrams were restored, supposing the lengths of the cords used to have been 5 feet and 9 feet, the diagrams became exactly similar, and, allowing 2 per cent. clearance, the expansion line came to be the true expansion line for that cut-off. The mean pressure was 14 per cent. larger than from the original diagram.

Such instances as these seemed to sufficiently establish a case against the blind faith which appeared to be at present placed in the accuracy of the indicator diagrams. But, in conclusion, the author stated that he should be very disappointed if anything in this investigation should have the effect of diminishing reliance on the indicator itself. He would have the instrument treated fairly, and instead of being the object of unthinking worship he would have it the object of careful study and experimental investigation, so that the limits of its wonderful perfection might be known exactly, and that reliance placed on it which sprang from knowledge.

\section{THE VISITATION OF THE ROYAL OBSERVATORY, GREENWICH}

THE visitation of the Royal Observatory took place on Saturday last, when, in spite of bad weather, there was a numerous attendance. The following extracts (condensed in some case $)$ from the Report of the Astronomer-Royal to the Board of Visitors indicate the work of the past year. It will be gratifying to all to knuw that a considerable increase in the optical power of the Observatory is in contemplation.

Transit-Circle.-A reversion-prism made by Messrs. Troughton and Simms has been used since last June in obse vations with the collimators as well as with the transit-circle to reverse the apparent direction of measurement or of motion, a movement towards the left (as in transits of south stars) being converted into a movement towa ds the right, or upwards, or downwards, according to the position of the plane of reflection of the reversion-prism. The collimation-observations show no sensible personality depending on the apparent direction of measurement ; it has, however, been considered well, in order to eliminate any possible effect of the kind, to take half the measures in each determination of collimation with the direction of movement of the wire reversed as regards right and left. In the transits the practice is to observe on each day two clock-stars and also circumpolar stars with the direction of motion reversed. A comparison of the results from the reversed and ordinary observations of clock-stars shows sensible differences in the case of some observers, who, however, have probably not yet settled down into a definite habit of observing stars which appear to move in the reverse direction.

In order to determine absolute personal equations in the observation of slow-moving as well as of quick-moving stars of various magnitudes (whether the motion be from right to left or the reverse) and of limbs of the sun, moon, or planets, the Astronomer Royal has arranged, in concert with Mr. Simms, a personal equation instrument to be used with the transit-circle. In this instrument, which is on the point of completion, and was seen at work on Saturday, a vertical plate with a circular aperture, 6 inches in diameter, to represent the sun or moon, and several small pinholes, to represent stars of different magnitudes, is placed in the focus of an object-glass of about 7 inches aperture and of about 50 feet focal length (which is attached to the dew-cap of the transit-circle, when horizontal and pointing north), and is carried smoothly by clockwork from east to west or west to east at a rate which may be varied at will from that of a very close circumpolar star to three or four times that of an equatorial star by an ingenious but simple mechanical contrivance devised by $\mathrm{Mr}$. Simms. The apertures in the vertical plate are illuminated by direct sunlight or moonlight reflected by a plane mirror towards the object-glass, and the times of transit of the artificial sun, moon, or stars, which are to be observed over the wires of the transit-circle, are also registered automatically on the chronograph by means of insulated platinum studs, corresponding to the artificial objects, which make contact with other studs, corresponding to the wires in the field of view of the transit-circle. 
Since last October transits of the close circumpolar stars have been taken at the middle wire set to successive revolutions of the R.A. micrometer, thus virtually introducing a system of very close equidistant wires for the slow-moving stars. It is thus found that a larger number of separate observations can be obtained in a moderate time, a point of special importance in changeable weather. The equality of suecessive intervals of the R.A. micrometer-screw was tested last January for each revolution through a range of twelve revolutions, and also for every tenth of the three middle revolutions, and the errors of the screw appear not to exceed the errors of observation. The determination was made by means of the south collimator, the eye-piece of the transit-circle having been turned through $90^{\circ}$. The observations of close circumpolar stars have also been discussed with a view of testing the equality of successive revolutions of the R.A. micrometer screw, the results being very satisfactory. The screws of the microscope-micrometers also were examined by means of the south collimator on March 27 and following days, successive intervals being measured for each revolution and third of a revolution from $-\mathbf{I}^{\mathrm{r}}$ to $6^{\mathrm{r}}$. Though there is evidence of considerable wear in the individual screws, which have been in constant use since 1875 , the methed of using them (the action of the spring being in opposite directions for the micrometers of each pair) entirely eliminates this effect from the mean, and the resulting errors (which are probably casual errors of observations) do not exceed $0^{\prime \prime} \cdot 05$ at any part of the screws.

The subjects of meridian observations in the past year have been as usual the sun, moon, planets, and fundamental stars, with other stars from a working catalogue, which now contains about 2,750 stars. About 380 stars have been lately added to the list from the "Harvard Photometry," with a view of making the forthcoming Greenwich Catalogue of stars down to the sixth magnitude as complete as possible. It is hoped that all of these stars will have been sufficiently observed by the end of 1886 , when it is proposed to form a Ten-Year Catalogue, epoch $1882^{\circ} \mathrm{O}$. The annual catalogue of stars observed in I884 contains about $\mathrm{I}, 370$ stars.

The following statement shows the number of observations with the transit-circle made in the twelve months ending 1885 , May 20:

Transits, the separate lim's being counted at separate $\begin{array}{lllllll}\text { observations... } & \ldots & \ldots & \ldots & \ldots & \ldots & 5523\end{array}$

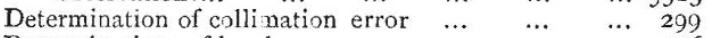

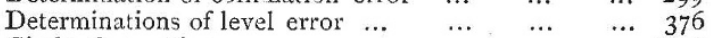

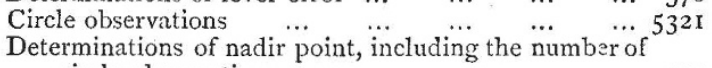
circle-observations

$\begin{array}{llll}\text { Reflection-observations of stars (similarly included) } & \ldots & 619\end{array}$

The discordance between the nadir observation and the mean of the results from reflection observations of stars north and south of the zenith has recently become very small, the correction dcduced for the first four months of the present year being only $-\mathrm{O}^{\prime \prime} \cdot 07$. The mean correction indicated by the observations of I 884 was $-\mathrm{O}^{\prime \prime} \cdot 36$, whilst those of 1883 gave the value $-0^{\prime \prime} \cdot 45$. The steady increase of this discordance from 1878 to 1883 and its subsequent decrease remained unexplained, no change having been introduced into the method of observation of the nadir point or of stars by reflection during the last two years.

The apparent flexure of the transit-circle, as found by means of the collimators, has again changed sign. From six determinations made on 1884 June 3, Sept. 9, Sept. 29, Oct. 5, Oct. 20, and May 20 (the reversion-prism being used on each occasion except the first) the resulting values (found by four different observers) are $-\mathrm{O}^{\prime \prime} \cdot 47,+\mathrm{I}^{\prime \prime} \cdot \mathrm{0O},+\mathrm{O}^{\prime \prime} \cdot \mathrm{O} 3,+\mathrm{O}^{\prime \prime} \cdot \mathrm{ro}$, and $+\mathrm{o}^{\prime \prime} \cdot 08$, the mean of which is $+\mathrm{O}^{\prime \prime} \cdot \mathrm{r} 7$, agreeing closely with the value $+\mathrm{O}^{\prime \prime} \cdot \mathrm{I} 3$ found by nine accordant determinations in the period 1879 to 1882 , whilst the mean of five determinations by three different observers in $1883 \mathrm{~g}$ ave the value $-\mathrm{O}^{\prime \prime} \cdot 49$. No correction for flexure (as distinct from the $\mathrm{R}-\mathrm{D}$ correction) has been applied to the observations since 1879 .

The correction for $\mathrm{R}-\mathrm{D}$, the error of assumed colatitude, and the position of the ecliptic, have been investigated for 1884 . The computation of the geocentric and heliocentric errors for the planetary results is not yet complete.

The correction for discordance between reflection and direct observations of stars, deduced from observations in 1884 which extend from Z.D. $69^{\circ}$ north to Z.D. $70^{\circ}$ south is $-0^{\prime \prime} \cdot 02+0^{\prime \prime} \cdot 66$ $\sin$ Z.D. The assumed formula $a+b \sin z$ represents the observations of 1884 satisfactorily throughout the whole range of zenith distance.

The value found for the colatitude from the observations of $\mathrm{r} 884$ is $38^{\circ} \cdot 3 \mathbf{I}^{\prime} \cdot 2 \mathbf{I}^{\prime \prime} \cdot 9 \mathrm{I}$, differing only by $\mathrm{O}^{\prime \prime} \cdot$ or from the assumed value; the correction to the tabular obliquity of the ecliptic is $+o^{\prime \prime} \cdot 57$; and the discordance between the results from the summer and winter solstices is - $0^{\prime \prime} \cdot 99$.

The mean error of the moon's tabular place (computed from Hansen's Lunar Tables with Prof. Newcomb's corrections) is $+0{ }^{\circ} \mathrm{O} 2$ in R.A. and $+\mathrm{O}^{\prime \prime} \cdot 29$ in longitude as deduced from IO4 meridian observations in 1884 .

Altazimuth. - The observations with this instrument have been restricted to the period from last quarter to first quarter in each lunation, the total number of observations of various kinds made in the I 2 months ending 1885 May 20 being as follows :-

$\begin{array}{llllll}\text { Azimuths of the moon and stars } & \ldots & \ldots & \ldots & \ldots & 32 \mathbf{I} \\ \text { Azimuths of the azimuth-mark } & \ldots & \ldots & \ldots & \ldots & \text { I81 } \\ \text { Azimuths of the collimating-mark } & \ldots & \ldots & \ldots & 192 \\ \text { Zenith-distances of the moon } & \ldots & \ldots & \ldots & \ldots & 178 \\ \text { Zenith-distances of the collimating-mark } & \ldots & \ldots & 196\end{array}$

Since last December a "reversion prism" has been used to reverse the apparent direction of motion in the observation with lamp right and in that with lamp left on alternate nights.

Clocks.-On Jan. I the public clock at the Otservatory entrance and the other mean solar clocks were put forward 12 hours so as to show Greenwich civil time, starting at midnight and reckoning from oh. to $24 \mathrm{~h}$., which would correspond with the universal time recommended by the Washington Conference. The change from astronomical to civil reckoning has also been made in all the internal work of the Observatory, and has been carried out without any difficuity. Greenwich civil time is found to be more convenient on the whole for the purposes of this Observatory, but its introduction into the printed astronomical observations has been deferred to allow time f.r. a general agreement amongst astronomers to be arrived at. It is proposed, however, to adopt the civil day without further delay in the printed magnetical results, thus reverting to the practice previous to 1848 , and making the time-reckoning harmonise with that used in the meteorological results, the reckoning from oh. to $24 \mathrm{~h}$. being for the future adopted in both cases.

Reflex Zenith Tube.-The observations of $\gamma$ Draconis for determination of the temperature correction have been continued, and about 45 transits over the 30 wires have been observed at temperatures ranging from $46^{\circ}$ to $72^{\circ}$. Seven transits of 9 Aurigæ were al:o observed last February at low temperatures ranging from $42^{\circ}$ to $56^{\circ}$.

Equatorials. - The work on the Lassell equatoreal has occupied a great deal of attention during the past year, a number of repairs and alterations having been required in order to get the instrument into proper working order. The driving clock, which was found to drive the instrument at only three-fifths of the proper speed, has been altered, a slow motion in R.A. (to be worked from the observing stage) has been contrived, a new slide of improved construction has been made for gearing the driving-screw into the hour-circle, the teeth of the hour-circle have been re-cut, a firm declination clamp has been applied, an improved edge suspension for the large mirror (consisting of a steel band which encircles the mirror and is supported by brackets at six equidistant points of the circumference) has been contrived, a new and firmer mounting of the small mirror has been made, and the ere-piece has been mounted firmly on a plate which allows it to be tilted in any direction, for optical adjustment. The framed ir $\mathrm{n}$ base which supports the instrument has been bricked up and filled with concrete, and this, with the other alterations, has greatly increased the stability of the telescope, which is now quite satisfactory. Difficulty is, however, still experienced from want of stability of the optical axis of the large mirror, which requires to be readjusted continually, as the telescope is moved. When the mirrors have been properly ad. justed the definition appears to be very good, the companion to Vega being shown with remarkable distinctness without any trace of scattered light from the large star.

The south-east and Sheepshanks equatorials are in good order, as also is the Simms' six-inch equatorial mounted in the south ground.

With one or more of these equatorials, or with the altazimuth, 30 occultations of stars by the moon (19 disappearances and I I reappearances, including 7 disappearances and 9 reappearances during the lunar eclipse of October 4), and 57 phenomena of 
Jupiter's satellites, have been observed in the twelve months ending I 885 May 20 , and the observations have all been completely reduced to the end of 1884 . Comet $(c)$ I 884 has been observed on four nights, the Lassell reflector or one of the other equatoreals being employed, and some measures of distances and position-angle of double stars, as well as a large number of observations fo: determining the value of $\mathrm{I}^{\text {rev }}$. of the screw in different parts of the field of view have been made with the Airy double-image micrometer mounted on the Sheepshanks' or Simms' equatorial.

Micrometer measures of some of the satellites of Saturn (including Enceladus) were made on seven nights with the Lassell equatorial.

Spactroscopic and Photograshic Observations. - The solar prominences have been observed with the half-prism spectroscope on only two days, the photographic reductions having pressed very severely on the spectroscopic assistant during the long continued maximum of sun-spots.

For the determination of motions of stars in the line of sight, 569 measures have been made of the displacement of the $\mathrm{F}$ line in the spectra of 47 stars, and 72 measures of the $b$ lines in 14 $\mathrm{s}^{\prime}$ ars, besides measures of the displacements of the $b$ and $\mathrm{F}$ lines in the spectra of the east and west limbs of Jupiter, and of the east and west ansæ of the rings of Saturn, and comparisons with lines in the spectrum of the moon, or of the sky, made in the course of each night's observations of star-motions, or on the following morning, as a check on the general accuracy of the results for star-motions. The observations of the last twelve months confirm the change in the motion of Sirius, which now appears to be approaching the sun at the rate of about 20 miles a second. As there is great difficulty in the use of a pointer or cross-wires for measuring both the broad dark line in the star's spectrum and the narrow bright comparison line, Mr. Maunder has suggested the use of a reversion spectro cope (on the double-image principle) for these observations, and Prof. Pritchard has kindly lent the reversion spectroscope of the Oxford University Observatory, in order that the suitability of that form of instrument may be tested. The spectroscopic observations of all kinds are completely reduced to the present time.

In the twelve months ending I 885 May 20 photographs of the sun have been taken on I 73 days, and of these $43 \mathrm{I}$ have been selected for preservation, the record being not so complete as usual, owing partly to the loss of several days during the adjustment of the instrument after the adaptation of the secondary magnifier, and partly to a failure of the supply of diy plates in July last during the absence of Mr. Maunder. There were only two days on which the sun's disk was observed to be free from spots.

The mean spotted area of the sun was slightly less in 1884 than in 1883 and slightly greater than in 1882 , whilst the faculæ in 1884 showed a slight increase as compared with 1883 , and a slight falling off as compared with $\mathbf{r} 882$. It would seem that the maximum both of sun-spots and faculæ occurred about the end of 1883 or beginning of 1884 .

For the year I884 Greenwich photographs are available for measurement on 152 dsys, and Indian photographs filling up the gaps in the series on 159 days, making a total of $31 \mathrm{I}$ days out of 366 on which photographs have been measured. In 1883 the total number of days was 340 , viz., Greenwich series 215 days, supplemented by Indian photographs received from the Solar Physics Co nmittee on 125 days.

Magnetic Instruments. - The following are the principal results for magnetic elements for 1884:Approximate mean westerly
declination

$18^{\circ} .8^{\prime}$.

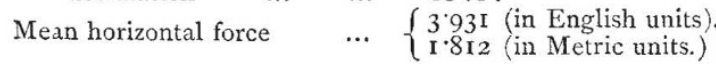

(67.29. 8 (by 9 -inch needles) $\{67.29 \cdot 32$ (by 6 -inch needles) (67.30. 9 (by 3 -inch needles).

In the year $18 S_{4}$ there were only five days of great magnetic disturbance, but there were also about 20 days of lesser disturbance for which it appears desirable to publish tracings of the photographic curves. It may be interesting to add the tracings for a few quiet and nearly quiet days in order to exhibit the shuracteristic; of the ordinary diurnal movement.

Commencing with 1883 the magnetic diurnal inequalities of declination, horizontal force, and vertical force have been discussed by the method of harmonic analysis, the harmonic expressions for these inequalities being obtained for each month and for the year with arguments expressed in apparent solar time as well as in mean solar time.

Meteorological Observations. - The mean temperature of the year 1884 was $50^{\circ} \%$, being $I^{\circ} 4$ higher than the average of the last 43 years. The highest air temperature (in the shade) was $94^{\circ} \cdot 1$ on Aug. II, and the low ast $24^{\circ} .5$ on Nov. 25. The mean monthly temperature was above the average excepting in the months of April, June, Oct. and Nov.

The mean daily motion of the air in 1884 was 286 miles, being 3 miles greater than the average of the last 17 years. The greatest daily motion was 891 miles on Jan. 23 , and the least 78 miles on Feb. 8. The only recorded pressure exceeding 20 lbs. on the square foot in 1884 was 22.7 lbs. on Jan. 23 , after which the connecting chain of the pressure plate broke, as mentioned in the last report. It is probable that greater pressures occurred afterwards on the same day, and also in the gale of Jan. 26, at which date the chain had not been renewed.

During the year I 884 Osler's anemometer showed an excess of about 25 revolutions of the vane in the positive direction $\mathrm{N}, \mathrm{E}, \mathrm{S}, \mathrm{W}, \mathrm{N}$, excluding the turnings which are evidently accidental.

The number of hours of bright sunshine recorded by Campbell's sunshine instrument during 1884 was I I I 5 , which is about Ioo hours less than the average of the seven preceding years. The aggregate number of hours during which the sun was above the horizon was 4465 , so that the mean proportion of sunshine for the year was $0^{\prime 25}$, constant suushine being represented by $\mathbf{I}$.

The rainfall in 1884 was. $18^{\circ} 0$ inches, being about 7 inches below the average of the last 40 years.

Chronometers and Time Signals. - The number of chronometers now being tested at the Observatory is $\mathrm{r}_{5} \mathrm{I}$, and of these I03 (79 box-chronometers, I3 pocket-chronometers, and II deck-watches) belong to the Navy, 40 are placed here for the annual competitive trial, and 8 are on trial for purchase by the Austrian Government.

The first six chronometers in the competitive trial of 1884 were rather above the average of the last ten years as inferred from the trial numbers. As much difficulty is experienced in maintaining the chronometer oven at a nearly constant temperature, an apparatus has been procured from Mr. Kullberg which is designed to effect this automatically, by the action of a compensation-bar, which, as the temperature rises, gradually closes a small hole through which the supply of gas to the gas burners passes. The apparatus has not yet been brought into use, as the chronometer oven has been constantly required for testing chronometers since it has been received.

The automatic drop of the Greenwich time-ball failed on 6 days through the clock-train stopping. The ball was not raised on 3 days on account of the violence of the wind.

As regards the Deal time-ball, which is now dropped by current passing through the chronopher of the Post Office telegraphs, there have been fourteen cases of failure owing to interruption of the telegraphic connections, and on one day the current was too weak to release the trigger without the assistance of the attendant.

In connection with the establishment of hourly time signals at the Start or Lizard, which was long advocated by Sir G. B. Airy, I have received from the Committee of I.loyds', in answer to my inquiry, an assurance that that corporation would be willing to undertake the maintenance of hourly time-signals at any of their signal-stations, provided the Government would supply the necessary apparatus. After consultation with Capt. Wharton, it has been thought better that, before taking further steps, some preliminary trials should be made of a collapsible cone as an hourly time-signal, faciiities for doing which exist at Devonport. As regards ball-drop or other time-signal, I would propose that it should be made automatically by a local clock, to be corrected daily by the help of a time-signal from Greenwich at ro a.m., which should automatically start an auxiliary seconds pendulum, suspended freely just behind the clock pendulum. The attendant would then accelerate or retard his clock pendulum (by electro-magnetic action as in the Greenwich mean solar clock) so as to make it pass through the middle of its vibration at the same time and in the same direction as the auxiliary pendulum, and thus to indicate accurately Greenwich mean time. A return signal to Greenwich sent by 
the local clock at the next hour (I I A.M.) would show that this clock had been properly corrected, and would be a guarantee for the general accuracy of the time-signals. Preliminary trials have shown that the observation of coincidence of vibration of two pendulums can be made with great certainty, and Messrs, E. Dent and Co. are now arranging for the mounting of an auxiliary pendulum on one of the transit of Venus clocks, and for adapting it to give hourly time-signals.

The errors of the Westminster clock have been under Is, on 50 per cent. of the days of observation, between Is. and 25 . on 29 per cent., between $2 \mathrm{~s}$. and $3 \mathrm{~s}$. on ro per cent., between $3 \mathrm{~s}$. and 4 s. on 7 per cent., and over 4 s. on 4 per cent.

During the past year the Observatory has lost the valuable services of Mr. Dunkin, who retired on August 25, after an honourable service of forty-six years, which has been throughout characterised by remarkable zeal and ability, and has contributed largely to maintain the credit of the Observatory. Mr. Dunkin has been succeeded in the post of Chief Assistant by $\mathrm{Mr}$. H. H. Turner, B.A., of Trinity College, Cambridge.

The report concludes as follows :-

During the past year the various classes of work carried on in this Observatory have been somewhat extended. The meridian observations are more numerous than usual, and various subsidiary investigations involving considerable labour have been undertaken with a view to increase their accuracy. A large number of spectroscopic determinations of star-motions have been obtained, and the long continued maximum of sunspots has made the photographic measurements and computations much heavier than in any previous year. Extensions have a'so been made in the magnetic and meteorological branch, which appeared very desirable, but which have pressed rather severely on Mr. Ellis and his staff.

Turning to the future, I wish to invite the attention of the visitors to the circumstance that an increase in our optical means is required to enable us to carry out satisfactorily the determinations of proper motions of stars in the line of sight with the spectroscope, a work which peculiarly belongs to this Observatory, as supplementing the determinations of proper motions from meridian observations.

The aperture of our largest refractor (1293 inches) is too small to allow of our observing successfully with the spectroscope any but the brightest stars, and though the Lassell reflector is somewhat more powerful, its mounting and clock-work are not adapted to carry a heavy spectroscope with the necessary steadiness and accuracy of motion. The firmness of the mounting of the south-east equatorial and the perfection of its clock-work would make it peculiarly suitable for this class of work if it carried a much larger object-glass.

After careful consideration of the conditions I have satisfied myself that an object-glass of 28 inches aperture and of 28 feet focal length could be mounted on the south-east equatorial, in place of the present object-glass of less than half that aperture ; and I hive ascertained that Mr. Grubb would be prepared to undertake the construction of such an object-glass with a tube suited to the special requirements of the case, so that the telescope would be equally available for eye-observation or for use with the spectroscope. With Mr. Grubb's assistance, I have prepared a model showing how this may be arranged.

While a large refractor is required specially for spectroscopic observations, it seems desirable also on other grounds that this Observatory should possess an equatorially mounted telescope comparable with those of other first-class observatories, so that we may no longer be prevented by deficient optical means from obtaining complete series of observations of comets and faint satellites.

\section{VIVISECTION}

A RETURN has been issued by the Home Office containing the reports of inspectors showing the number of experiments performed on living animals during the year 1884 under licences granted according to the Act 39 and 40 Victoria, c. 77, distinguishing painless from painful experiments.

The former of the two reports deals with England and Scotland, the latter with Ireland. They are as follows :-

"(I) The names of the 49 persons who held licences during any part of the year are given in the suljjoined tables, in one of which are entered the names of those licensces who performed any experiments, 34 in number; and, in the other, the names of those who performed none.
" (2) The total number of experiments of all kinds performed during the year was about $44 \mathrm{I}$.

"Of these, I40 were done under the restrictions of the licence alone, 78 under the same restrictions, but under certificates in column I (lecture illustrations); $\mathbf{1} 45$ under certificates in column 2; 76 under those in column 3 ; and 2 under a certificate in column 4.

"(3) With regard to the infliction of pain, as in all the experiments, except those under special certificates in columns 2 , 3 , and 4 , the animals are rendered insensible during the whole of the experiment, and are not allowed to recover consciousness, no appreciable suffering would be caused if the provisions of the Act are faithfully carried out, as there is not the least reason to doubt they were.

"With respect to experiments under certificates in columns 2, 3 , and 4 , which dispense either wholly or partially with the use of anæsthetics, it should be stated :-

" $(a)$ That of the 145 experiments performed under certificates in column 2, 99 consisted in simple inoculation with a morbid virus, in which no operation beyond the prick of a needle was required, and for which the administration of an anæsthetic would only have entailed needless annoyance and distress to the animal. In these experiments any appreciable suffering would be felt only in those cases in which the inoculation took effect, involving about the same amount of pain as ensues on ordinary vaccination, before the brief period the animals were allowed to survive. Of such cases, according to the returns I have received, about 16 occurred. Of the remaining 46 experiments under these certificates, 24 were performed for the purpose of medicolegal inquiries in cases of suspected poisoning, resulting in the death by tetanus of three frogs and six mice, which survived, however, only a few minutes; ro other cases under the same head were experiments on the infection of fish with a species of fungus, very destructive in certain rivers and streams; and five on the effects of immersion of fish in distilled water, which proved fatal to about thirty minnows and sticklebacks. In none of these cases could it be said that any appreciable suffering was inflicted. In seven cases, in which salts of ammonia were hypodermically injected, two are returned as having suffered pain, but of a very trifling character.

“( $(b)$ Of the 76 experiments under certificates in column 3, 47 required a simple operation, but this being done under anæsthesia, was unfelt, and the after-effects, though in many of the cases resulting in partial paralysis, are reported as having been unattended with actual pain in any case. The remaining 29 were by simple inoculation, and none were attended with pain.

"(4) In conclusion, therefore, it may be stated that the amount of direct or indirect actual suffering, as the result of physiological and therapentical experiments performed in England and Scotland, under the Act in the year 1884, was wholly insignificant.

"The Right Hon. the Secretary of State." "GEORGE Busk, Inspector

“ 16, Harcourt Street, Dublin, May 17

"SIR,--In accordance with your instructions I beg to submit the following table, showing the licences in force in Ireland during the year 1884 under the Act 39 and 40 Vict., c. 77 . No certificate has been allowed during the year.

"Several of the licences in force during the previous year have expired, and renewals have not been sought for.

"Under the licences in force thirteen experiments have been made; they were all painless. I am of opinion that the experiments in question were useful ones; eleven of them were intended to elucidate the actions of drugs, and the remaining two to assist the investigation of certain circulatory phenomena which have a bearing upon the treatment of disease.

$$
\text { "I have, \&c., }
$$

"W. THORNLEY STOKER

"To the Right Hon. the Chief Secretary for Ireland." In each case the report is followed by a list of all persons who hold such licences, the places where they are permitted to make experiments, and the nature of the certificate held.

\section{UNIVERSITY AND EDUCATIONAL INTELLIGENCE}

CAMBRIDGE.-The Annual Report of the Museums and Lecture-Rooms Syndicate, recently published, contains the 